Zumindest sollte jeweils eine Kandidatin und ein Kandidat für das Kollegium der Europäischen Kommission benannt werden.

\section{Völkerrecht}

Der Staatenbericht der Bundesregierung wird Mitte 2014 an den CEDAW-Ausschuss übermittelt werden. Es ist davon auszugehen, dass er die im Koalitionsvertrag vorgesehenen Neuausrichtungen u.a. im Hinblick auf Frauen in Führungspositionen berücksichtigt. Der Staatenbericht wird von der Zivilgesellschaft - auch vom djb - eingehend geprüft und im Schattenbericht kommentiert werden.
Der djb begrüßt, dass die Gleichstellung von Frauen und Männern und die Durchsetzung der Rechte von Mädchen und Frauen zu einer Querschnittsaufgabe deutscher Entwicklungszusammenarbeit werden sollen. Konsequent sollte sich die Bundesregierung daher auch dafür einsetzen, dass die Geschlechtergleichheit als Querschnittsklausel in die Nachhaltigkeitsziele (SDG) 2015 aufgenommen wird und die einzelnen Politikfelder eine Geschlechterperspektive enthalten.

\section{Sabine Overkämping}

Vorsitzende

\title{
Unmittelbare Entgeltdiskriminierung durch betriebliche Praxis
}

\section{Ein neues Urteil wirft Fragen auf}

\section{Dr. Karin Tondorf}

Mitglied der djb-Kommission Arbeits-, Gleichstellungs- und Wirtschaftsrecht; Forschung \& Beratung zu Entgelt- und Gleichstellungspolitik, www.karin-tondorf.de, Seddiner See

Dass Frauen wegen Entgeltdiskriminierung klagen, ist in Deutschland höchst selten. Eine Werkstattleiterin einer Universität hat es kürzlich gewagt, weil sie bei gleicher Arbeit wesentlich schlechter eingruppiert ist als ihr männlicher Kollege. Ihre Klage wurde in der zweiten Instanz abgewiesen. ${ }^{1}$ Die Begründung: Sie habe kein geschlechtsbezogenes Diskriminierungsmotiv des Arbeitgebers glaubhaft machen können. Das Gericht hielt vielmehr die Gründe des Arbeitgebers für „nachvollziehbar“, der das rechtliche Risiko einer Änderungskündigung des begünstigten Mannes vermeiden wollte. „Damit fehlt es an einem Indiz dafür, dass die Aufrechterhaltung der höheren Eingruppierung wegen des Geschlechts erfolgt ist.", heißt es in der Urteilsbegründung. Obwohl sich das Gericht mit grundsätzlichen Fragen des unionsrechtlichen Entgeltgleichheitsgebotes (Art. 157 AEUV) befasst hat, wurde eine Revision zum BAG nicht zugelassen.

Die in der Entscheidung vertretene Rechtsauffassung wirft Fragen auf, die über diesen Einzelfall hinausweisen: Wie kann ein Verdacht auf unmittelbare Entgeltdiskriminierung aufgrund des Geschlechts glaubhaft gemacht und der Rechtsanspruch des gleichen Entgelts für gleiche Arbeit wirksam durchgesetzt werden? Die Klärung dieses Aspekts dürfte insbesondere auch weibliche Führungskräfte interessieren, die z.B. als Nachfolgerin eine Stelle besetzen, die vorher ein Mann innehatte, jedoch schlechter bezahlt werden. Aber auch Frauen in mittleren und unteren Entgeltgruppen könnten betroffen sein. Prüfungen der Entgeltgleichheit mit eg-check.de ${ }^{2}$ zur betrieblichen Eingruppierungspraxis weisen jedenfalls darauf hin, dass Tätigkeiten von Frauen und Männern nicht immer gleich eingruppiert sind. Unmittelbare Entgeltdiskriminierung aufgrund des Geschlechts es gibt sie offenbar noch. Zwar nicht durch eindeutige und schnell identifizierbare Regelungen in Tarifverträgen, wohl aber durch eine schwer durchschaubare, tabuisierte betriebliche Entgeltpraxis.

\section{Diskriminierungsverdacht glaubhaft machen}

In dem angesprochenen Fall forderte das Gericht von der Klägerin, Anhaltspunkte dafür zu liefern, dass der Arbeitgeber sie als Frau diskriminieren wollte: „Erforderlich ist, dass die vorgetragenen Tatsachen aus objektiver Sicht mit überwiegender Wahrscheinlichkeit darauf schließen lassen, dass die weniger günstige Behandlung durch ein verpöntes Merkmal motiviert ist. " 3 Ein geschlechtsdiskriminierendes Motiv des Arbeitgebers durch Tatsachen darzulegen, dürfte für betroffene Frauen objektiv schwierig sein. Nur naive Arbeitgeber dürften sich offen zu entsprechenden Äußerungen oder Verhaltensweisen hinreißen lassen. Wollte ein Arbeitgeber bewusst diskriminieren, wäre Schweigen die klügere Alternative. An dem Fehlen des Nachweises eines Diskriminierungsmotivs darf die Rechtsdurchsetzung der Entgeltgleichheit jedoch nicht scheitern. Aus unionsrechtlicher Sicht ist der Nachweis, dass das Geschlecht ursächlich für eine Benachteiligung ist, nicht erforderlich. ${ }^{4} \mathrm{Nach}$ der Rechtsprechung des $\mathrm{EuGH}^{5}$ ist es Sache der Klägerin „zu beweisen, dass die Beklagte ihr ein niedrigeres Entgelt zahlt als ihrem zum Vergleich herangezogenen männlichen Kollegen und dass sie tatsächlich die gleiche oder eine gleichwertige, mit dessen Arbeit vergleichbare Arbeit verrichtet, so dass sie dem ersten Anschein nach Opfer einer nur mit dem unterschiedlichen Geschlecht erklärbaren Diskriminierung ist.“ Dieser Beweis wurde im Klagefall erbracht.

LAG Baden-Württemberg, Az: 1 Sa 7/13 vom 21.10.2013.

Nähere Ausführungen hierzu: www.eg-check.de.

S. Fußnote 1, S. 13.

4 Siehe auch Winter, Regine, 1998: Gleiches Entgelt für gleichwertige Arbeit. Ein Prinzip ohne Praxis. Baden-Baden, S. 236.

5 EuGH v. 26.6.2001 C-381/99 „Brunnhofer“, Rn. 58. 


\begin{tabular}{|l|l|}
\hline \multicolumn{2}{|l|}{ Unmittelbare und mittelbare Diskriminierung } \\
\hline Richtlinie 2006/54/EG, & Richtlinie 2006/54/EG, Art. 2 (1) b) \\
Art. 2 (1) a) „unmittelbare & "mittelbare Diskriminierung“: Eine \\
Diskriminierung“: Eine & Situation, in der dem Anschein nach \\
Situation, in der eine & neutrale Vorschriften, Kriterien oder \\
Person aufgrund ihres & Verfahren Personen des einen Ge- \\
Geschlechts eine weniger & schlechts in besonderer Weise gegen- \\
günstige Behandlung & über Personen des anderen Geschlechts \\
erfährt, als eine andere & benachteiligen können, es sei denn, die \\
Person in einer vergleich- & betreffenden Vorschriften, Kriterien oder \\
baren Situation erfährt, & Verfahren sind durch ein rechtmäßiges \\
erfahren hat oder erfahren & Ziel sachlich gerechtfertigt und die \\
würde. & Mittel sind zur Erreichung dieses Ziels \\
& angemessen und erforderlich. \\
\hline
\end{tabular}

$\Delta$ Abb. 1: Keine Rechtfertigung bei unmittelbarer Entgeltdiskriminierung.

Sodann obläge es dem Arbeitgeber zu beweisen, dass nicht gegen den Grundsatz des gleichen Entgelts verstoßen wurde. ${ }^{6}$ Bei unmittelbarer Entgeltungleichbehandlung gibt es - anders als bei mittelbarer - keine Möglichkeit der Rechtfertigung. ${ }^{7}$ Dies zeigt schon die unterschiedliche Wortwahl bei den Definitionen der beiden Diskriminierungsformen (s. Abb. 1). Daher kann sich der Arbeitgeber nur durch arbeitsbezogene Gründe entlasten, indem er nachweist, „dass die von den beiden betroffenen $\mathrm{Ar}$ - beitnehmern tatsächlich ausgeübten Tätigkeiten in Wirklichkeit nicht vergleichbar sind." 8

Dass im Ausgangsfall gleiche Arbeit verrichtet wurde, war für den in Frage stehenden Zeitraum der Forderung gar nicht strittig. Ob der Besitzstand des Werkstattleiters diskriminierungsfrei zustande kam, wurde seitens des Gerichts nicht geprueft.

Unverständlich ist auch die abschließende Wertung des Gerichts, dass das beklagte Land „aus nachvollziehbaren Gründen“ keine Absenkung des Entgeltes des begünstigten Mannes vornehmen wollte. Um die Schlechterstellung des Kollegen ging es der Klägerin jedoch gar nicht. Sie verlangte die „Angleichung nach oben “, was im Streitfall die übliche Rechtsfolge ist. ${ }^{9}$ Inwieweit dem Arbeitgeber zuzumuten ist, die Ungleichbehandlung durch eine höhere Bezahlung der Frau zu beseitigen, wurde nicht erörtert.

\footnotetext{
6 EuGH v. 26.6.2001 C-381/99 „Brunnhofer“, Rn. 60.

7 EuGH vom 18.5.2006 Rechtssache C-17/05 „Cadman“, Schlussanträge des Generalanwalts, Randnr. 24.

8 EuGH v. 26.6.2001 C-381/99 „Brunnhofer“, Rn. 61.

9 EuGH vom 15. 1. 1998 - Rs. C-15/ 96 "Schöning-Kougebetopoulou“, Leitsatz 2, siehe auch EuGH vom 31.5.1995 Rs. C-400/93 „Royal Copenhagen“, Leitsatz 5.
}

\section{Rezension:}

\section{Geschlechtergerechtigkeit steuern - Perspektiven- wechsel im Steuerrecht}

Ulrike Spangenberg / Maria Wersig (Hrsg.),

Geschlechtergerechtigkeit steuern - Perspektivenwechsel im Steuerrecht, Hochschule für Wirtschaft und Recht

Berlin 2013, 257 Seiten, 17,90 EUR

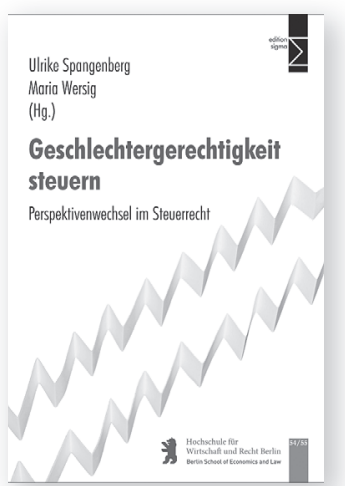

Aufnahme einer Erwerbstätigkeit für (Ehe-) Frauen unattraktiv zu machen (EC Commision, in: Bulletin of the International Bureau of Fiscal Documentation, No. 39, S. 62-66). 30 Jahre nach der EU-Studie wenden die Mehrzahl der europäischen Länder den Grundsatz der Individualbesteuerung an. Lediglich Deutschland, Frankreich, Irland, Luxemburg, Portugal und Spanien veranlagen Ehepaare grundsätzlich oder optional gemeinsam.

In Deutschland ist die Debatte um Geschlechtergerechtigkeit im Steuerrecht weitgehend auf die Besteuerung von Ehe und Familie beschränkt. In der öffentlichen Diskussion stehen der progressive Steuertarif bzw. die Höhe der Spitzensteuersätze. Die tatsächliche Steuerbelastung hängt jedoch von sehr viel mehr Faktoren ab. Beispielhaft zu nennen sind Werbungskosten- und Betriebsausgabenabzüge sowie Steuersubventionen. Die entsprechenden Regelungen sind zwar geschlechtsneutral formuliert, wirken aber tatsächlich sehr unterschiedlich - in Abhängigkeit von verfügbaren finanziellen oder zeitlichen Ressourcen, von Rollenverständnissen, von Arbeitsmarktstrukturen etc. Infolgedessen beeinflussen Ausgestaltung und Änderung des Steuersystems auch direkt die Lebensverhältnisse von Frauen und Männern. Vor allem in den USA und in Kanada ist die Diskussion um Geschlechtergerechtigkeit im Steuersystem sehr viel weiter fortgeschritten als in Deutschland. ${ }^{1}$ Mit dem vorliegenden Tagungsband werden - soweit ersichtlich - erstmals die

1 Nachweise ebenda, S. 9. 